\title{
Precisão experimental de ensaios de trigo analisados pelo método de Papadakis
}

\author{
Experimental precision in wheat cultivars trials using the Papadakis method
}

\author{
Lindolfo Storck ${ }^{\mathrm{I}}$ Marcio Só e Silva ${ }^{\mathrm{II}}$
}

RESUMO

O objetivo deste trabalho foi verificar se o uso da análise dos resultados dos ensaios de valor de cultivo e uso (VCU) de trigo pelo método de Papadakis modifica os pressupostos do modelo matemático e as medidas de precisão experimental. Para isso, foram usados os dados de produtividade de grãos de trigo de 48 ensaios de VCU, conduzidos sob delineamento de blocos ao acaso, entre 2008 e 2011. Para cada ensaio, sem e com o uso do método de Papadakis, foram realizados a verificação dos pressupostos, a análise de variância, os testes de hipóteses e calculadas as estatísticas para a identificação da precisão experimental. Esses pressupostos não foram violados para os dois métodos de análise (sem e com Papadakis). Com o uso do método de Papadakis (parcelas vizinhas), aumenta o índice de diferenciação de Fasoulas, 29,7 contra 15,5 sem o uso do método e a acurácia seletiva, 0,95 contra 0,90 sem o uso do método. Ensaios com três repetições, analisados com o método de Papadakis, possibilitam a identificação de cultivares de trigo com superioridade na produtividade de grãos com 90\% de precisão. Para manter a precisão de 90\% na análise sem o uso de Papadakis, seriam necessárias seis repetições.

Palavras-chave: pressupostos da anova, medidas de precisão, análise espacial, Triticum aestivum.

\section{ABSTRACT}

The aim of this research was to verify whether the use of wheat yield trials results analysis, using the Papadakis method, modifies the assumptions of the mathematical model and indicators of experimental precision. Wheat productivity data, 2008 to 2011 years, obtained from 48 cultivar yield trials under randomized block design were considered. In each trial, either with or without the use of the Papadakis method, the assumptions, analysis of variance and hypothesis tests were verified as well as the calculations to identify the experimental precision. The assumptions were not violated by both methods (with or without de Papadakis method). With the Papadakis method (neighboring plots) the differentiation of the Fasoulas index increased, 29.7 compared to 15.5 without the use of the method, and the selective accuracy, 0.95 against 0.90 without the method. Trials using three replications when analysed by the Papadakis method allowed to identify superior bean varieties with $90 \%$ precision. In order to maintain the same precision (90\%), six replications would be necessary without the Papadakis method.

Key words: anova assumptions, precisions measure, spatial analysis, Triticum aestivum.

\section{INTRODUÇÃO}

É conhecido que o planejamento experimental quanto à escolha adequada do delineamento experimental, do número de repetições, do tamanho de parcela, do manejo de pragas, doenças e plantas daninhas são procedimentos indispensáveis para a execução de ensaios com precisão experimental de magnitude aceitável (STEEL et al., 1997; RAMALHO et al., 2005).

As estatísticas acurácia seletiva, coeficiente de herdabilidade, coeficiente de determinação e valor do teste $\mathrm{F}$ para cultivares têm relação algébrica entre si, são mais adequadas do que o coeficiente de variação e a diferença mínima significativa para se avaliar a precisão experimental em ensaios de competição de híbridos de milho (CARGNELUTTI FILHO \& STORCK, 2007 e 2009). Usando os dados da produtividade dos ensaios de competição de cultivares de feijão e de soja, CARGNELUTTI

'Programa de Pós-graduação em Agronomia, Universidade Federal de Santa Maria (UFSM), Avenida Roraima 1000, Bairro Camobi, 97105-900, Santa Maria, RS, Brasil. E-mail: lindolfo@pq.cnpq.br. Autor para correspondência.

IIEmbrapa Trigo, Passo Fundo, RS, Brasil. 
FILHO et al. (2009) concluíram que os limites de classe de precisão estabelecidos, a partir das medidas de acurácia seletiva, do coeficiente de herdabilidade, do coeficiente de determinação e do valor do teste $\mathrm{F}$ para cultivares são adequados. Visando à melhoria da precisão experimental, estudos referentes ao planejamento experimental (tamanho de parcela e número de repetições) para ensaios de trigo foram executados (LORENTZ et al., 2007; HENRIQUES NETO et al., 2004). No entanto, esses resultados são insuficientes para subsidiar um planejamento adequado de uma rede de ensaios de avaliação de cultivares de trigo, pelo fato de não incluir a variação ambiental da região tritícola.

Dentre as formas de análise espacial (COSTA et al., 2005), o método de Papadakis consiste no ajuste dos valores obtidos nas parcelas em função das médias dos erros experimentais entre as parcelas vizinhas, usada como covariável, com a finalidade de diminuir a variância do erro experimental. A covariável mais adequada é a estimada em função do erro das parcelas de referência e as mais próximas (lado esquerdo, direito, frente e fundo) (CARGNELUTTI FILHO et al., 2003). O uso do método de Papadakis mostrou-se eficiente para a melhoria dos indicadores de precisão experimental em ensaios de soja (STORCK et al., 2008), milho (CARGNELUTTI FILHO et al., 2003; STORCK et al., 2010) e feijão (STORCK et al., 2011).

A magnitude da eficiência do método de Papadakis e a sua influência sobre os pressupostos (normalidade e independência do erro, homogeneidade de variância entre cultivares e a aditividade do modelo), para ensaios com diferentes cultivares de trigo, executados em diferentes locais, anos e épocas ainda não é conhecida. $\mathrm{E}$, assim como já foi constatado que, nos ensaios com as culturas da soja, milho e feijão, o método de Papadakis foi eficiente, este pode ser um procedimento adequado para a melhoria da precisão experimental dos ensaios de avaliação da produtividade de grãos de trigo.

O objetivo deste trabalho foi verificar se o uso da análise dos resultados dos ensaios de valor de cultivo e uso de trigo pelo método de Papadakis modifica os pressupostos do modelo matemático e as medidas de precisão experimental.

\section{MATERIAL E MÉTODOS}

Foram utilizados os dados de produtividade de grãos de trigo (Triticum aestivum) obtidos em 48 ensaios de valor de cultivo e uso (VCU), coordenados e executados pela Embrapa Trigo - Passo Fundo, RS.
O número de cultivares por ensaio variou entre 27 e 55 e o delineamento experimental adotado foi o de blocos ao acaso, com três repetições e parcelas úteis de $5,0 \mathrm{~m}^{2}$. Os 11 locais e os respectivos anos e número de cultivares por ensaio estão descritos na tabela 1 . Os dados de produtividade de grãos, de cada ensaio, foram submetidos à análise de variância, com testes de hipóteses (valor-p=0,05) para blocos e cultivares. Foram estimadas a eficiência relativa (ER) do uso de blocos, a média por cultivar, a amplitude entre as médias das cultivares e a média geral $(\hat{\mathrm{m}})$. Também foram estimados o coeficiente de variação do erro experimental $(\mathrm{CV})$ e a diferença mínima significativa entre as cultivares pelo teste de Tukey (valor- $p=0,05$ ) (D), $\mathrm{D}=\mathrm{q}_{\left(\alpha ; \mathrm{I} ; \mathrm{GL}_{\mathrm{E}}\right)} \sqrt{\mathrm{QM}_{\mathrm{E}} / \mathrm{J}}$, na qual $\mathrm{q}_{\left(\alpha ; \mathrm{I} ; \mathrm{GL}_{\mathrm{E}}\right)}$ é o valor da tabela para o teste de Tukey, I é o número de cultivares, $\mathrm{GL}_{\mathrm{E}}$ são os graus de liberdade do erro, $\mathrm{QM}_{\mathrm{E}}$ é a estimativa do erro experimental e J é o número de repetições (blocos). A diferença mínima significativa entre as médias de cultivares, pelo teste de Tukey, expresso em percentagem da média (DMS), foi obtida por $\mathrm{DMS}=100 \mathrm{D} / \hat{\mathrm{m}}$. Para avaliar a precisão experimental, foi obtido o índice de diferenciação de Fasoulas (IDF) (FASOULAS, 1983), estimado pela expressão $\operatorname{IDF}=200 \sum_{\mathrm{i}=1}^{\mathrm{n}} \mathrm{m}_{\mathrm{i}} /[\mathrm{n}(\mathrm{n}-1)]$, em que $\mathrm{n}$ é o número de cultivares do ensaio e $\mathrm{m}_{\mathrm{i}} \mathrm{o}$ número de médias que a i-ésima cultivar supera estatisticamente, após a aplicação do teste de Tukey.

Determinou-se o valor da estatística $\mathrm{R}^{2}$, designada por coeficiente de determinação (CARGNELUTTI FILHO \& STORCK, 2007), expressa por $\mathrm{R}^{2}=\mathrm{QM}_{\mathrm{c}} /\left(\mathrm{QM}_{\mathrm{c}}+\mathrm{QM}_{\mathrm{E}}\right)$, em que $\mathrm{QM}_{\mathrm{c}}$ é o quadrado médio de cultivar. Ainda, a acurácia seletiva (AS) foi estimada como sendo: AS $=$ (1$1 / \mathrm{F})^{1 / 2}$, em que $\mathrm{F}$ é o valor do teste $\mathrm{F}$ para cultivar (RESENDE \& DUARTE, 2007; CARGNELUTTI FILHO \& STORCK, 2009).

Tendo-se considerado as avaliações em cada bloco como medições realizadas no mesmo indivíduo (cultivar), estimou-se o coeficiente de correlação intraclasse para cultivares ou coeficiente de repetibilidade (método da análise de variância): $\hat{\rho}_{\mathrm{c}}=\hat{\sigma}_{\mathrm{c}}^{2} /\left(\hat{\sigma}^{2}+\hat{\sigma}_{\mathrm{c}}^{2}\right)$, em que $\hat{\sigma}_{\mathrm{c}}^{2}=\left(\mathrm{QM}_{\mathrm{c}}-\mathrm{QM}_{\mathrm{E}}\right) / \mathrm{J}$

e $\quad \hat{\sigma}^{2}=\mathrm{QM}_{\mathrm{E}}$. O número mínimo de medições (repetições $=$ blocos $=\mathrm{J}$ ) necessário para predizer o valor real dos indivíduos (cultivares), com base na determinação ou precisão pré-estabelecida $\left(\mathrm{R}^{2}\right)$, foi obtido conforme CRUZ \& REGAZZI (1997), ou seja, o valor de J, para um dado $R^{2}$, é: $J\left(R^{2}\right)=\left(1-\widehat{\rho}_{c}\right) R^{2} / \widehat{\rho}_{c}\left(1-R^{2}\right)$.

Foram estimados os erros de cada unidade experimental, tendo-se considerado o delineamento 
Tabela 1 - Número de genótipos de trigo (NG), número de filas (F) e colunas (C) do croqui experimental usado em diferentes locais, anos, épocas e categoria de ensaios.

\begin{tabular}{|c|c|c|c|c|c|c|c|}
\hline Ens & Local & Ano & Época & Categoria & NG & $\mathrm{F}$ & $\mathrm{C}$ \\
\hline 1 & Abelardo Luz, SC & 2010 & 1 & VCU Precoce & 50 & 3 & 50 \\
\hline 2 & Campos Novos, SC & 2008 & 1 & VCU Precoce & 32 & 3 & 32 \\
\hline 3 & Canoinhas, SC & 2009 & 1 & VCU Precoce & 27 & 3 & 27 \\
\hline 4 & Canoinhas, SC & 2010 & 1 & VCU Precoce & 55 & 6 & 28 \\
\hline 5 & Canoinhas, SC & 2011 & 1 & VCU Precoce & 27 & 4 & 21 \\
\hline 6 & Canoinhas, SC & 2011 & 1 & VCU Médio & 32 & 5 & 21 \\
\hline 7 & Chapecó, SC & 2008 & 1 & VCU Precoce & 32 & 3 & 32 \\
\hline 8 & Chapecó, SC & 2009 & 1 & VCU Precoce & 27 & 3 & 27 \\
\hline 9 & Chapecó, SC & 2011 & 1 & VCU Precoce & 27 & 4 & 21 \\
\hline 10 & Chapecó, SC & 2011 & 1 & VCU Médio & 32 & 4 & 24 \\
\hline 11 & Passo Fundo, RS & 2008 & 1 & VCU Precoce & 32 & 3 & 32 \\
\hline 12 & Passo Fundo, RS & 2008 & 2 & VCU Precoce & 32 & 3 & 32 \\
\hline 13 & Passo Fundo, RS & 2009 & 1 & VCU Precoce & 27 & 3 & 27 \\
\hline 14 & Passo Fundo, RS & 2009 & 2 & VCU Precoce & 28 & 6 & 14 \\
\hline 15 & Passo Fundo, RS & 2010 & 1 & VCU Precoce & 55 & 3 & 55 \\
\hline 16 & Passo Fundo, RS & 2010 & 2 & VCU Precoce & 55 & 3 & 55 \\
\hline 17 & Passo Fundo, RS & 2011 & 1 & VCU Precoce & 27 & 3 & 27 \\
\hline 18 & Passo Fundo, RS & 2011 & 1 & VCU Médio & 32 & 3 & 32 \\
\hline 19 & Passo Fundo, RS & 2011 & 2 & VCU Precoce & 27 & 3 & 27 \\
\hline 20 & Passo Fundo, RS & 2011 & 2 & VCU Médio & 32 & 3 & 32 \\
\hline 21 & Piratini, RS & 2010 & 1 & VCU Precoce & 50 & 3 & 50 \\
\hline 22 & Ponta Grossa, PR & 2011 & 1 & VCU Precoce & 27 & 8 & 11 \\
\hline 23 & Ponta Grossa, PR & 2011 & 1 & VCU Médio & 32 & 10 & 11 \\
\hline 24 & São Borja, RS & 2008 & 1 & VCU Precoce & 32 & 3 & 32 \\
\hline 25 & São Borja, RS & 2008 & 2 & VCU Precoce & 32 & 6 & 16 \\
\hline 26 & São Borja, RS & 2009 & 1 & VCU Precoce & 27 & 3 & 27 \\
\hline 27 & São Borja, RS & 2009 & 2 & VCU Precoce & 27 & 3 & 27 \\
\hline 28 & São Borja, RS & 2010 & 1 & VCU Precoce & 50 & 3 & 50 \\
\hline 29 & São Borja, RS & 2010 & 2 & VCU Precoce & 55 & 5 & 33 \\
\hline 30 & São Borja, RS & 2011 & 1 & VCU Precoce & 27 & 3 & 27 \\
\hline 31 & São Borja, RS & 2011 & 1 & VCU Médio & 32 & 3 & 32 \\
\hline 32 & São Borja, RS & 2011 & 2 & VCU Precoce & 27 & 4 & 21 \\
\hline 33 & São Borja, RS & 2011 & 2 & VCU Médio & 32 & 3 & 32 \\
\hline 34 & Três de Maio, RS & 2008 & 1 & VCU Precoce & 32 & 3 & 32 \\
\hline 35 & Três de Maio, RS & 2008 & 2 & VCU Precoce & 32 & 6 & 16 \\
\hline 36 & Três de Maio, RS & 2009 & 1 & VCU Precoce & 27 & 3 & 27 \\
\hline 37 & Três de Maio, RS & 2009 & 2 & VCU Precoce & 27 & 6 & 14 \\
\hline 38 & Três de Maio, RS & 2010 & 1 & VCU Precoce & 50 & 4 & 38 \\
\hline 39 & Três de Maio, RS & 2010 & 2 & VCU Precoce & 55 & 3 & 55 \\
\hline 40 & Três de Maio, RS & 2011 & 2 & VCU Precoce & 27 & 4 & 21 \\
\hline 41 & Três de Maio, RS & 2011 & 2 & VCU Médio & 32 & 4 & 24 \\
\hline 42 & Vacaria, RS & 2008 & 1 & VCU Precoce & 32 & 3 & 32 \\
\hline 43 & Vacaria, RS & 2010 & 1 & VCU Precoce & 55 & 3 & 55 \\
\hline 44 & Vacaria, RS & 2011 & 1 & VCU Precoce & 27 & 4 & 21 \\
\hline 45 & Vacaria, RS & 2011 & 1 & VCU Médio & 32 & 4 & 24 \\
\hline 46 & Victor Graeff, RS & 2010 & 1 & VCU Precoce & 55 & 3 & 55 \\
\hline 47 & Victor Graeff, RS & 2011 & 1 & VCU Precoce & 27 & 4 & 21 \\
\hline 48 & Victor Graeff, RS & 2011 & 1 & VCU Médio & 32 & 4 & 24 \\
\hline
\end{tabular}

Ciência Rural, v.44, n.3, mar, 2014. 
de blocos ao acaso (valor observado menos a média geral, menos os efeitos de bloco e de cultivar). Usando os valores do erro estimado, foi aplicado o teste de Lilliefors (SPRENT \& SMEETON, 2007) para verificar o pressuposto de normalidade. Para o teste da aleatoriedade dos erros, ou teste de sequência, a contagem do número de sequências de erros de mesma classe (acima ou abaixo da média) foi executada seguindo um caminhamento entre as colunas das diferentes filas de forma pré-estabelecida (segue por uma fila e volta pela outra). A estatística do teste da sequência é igual ao número de vezes em que se observa a troca de classe no caminhamento estabelecido para as parcelas experimentais. Esta estatística foi testada pela aproximação da distribuição normal padrão (SPRENT \& SMEETON, 2007). O teste de Bartlett (STEEL et al., 1997) foi aplicado para verificar a homogeneidade das variâncias dos erros entre as cultivares. A aditividade do modelo matemático foi verificada pelo teste de não-aditividade do modelo matemático de Tukey (SNEDECOR \& COCHRAN, 1989). As hipóteses das pressuposições (normalidade, aleatoriedade, homogeneidade e aditividade) foram testadas com valor- $\mathrm{p}=0,05$.

Adicionalmente, foram estimados os erros, tendo-se considerado o delineamento inteiramente casualizado (valor observado menos a média geral e menos o efeito de cultivar). Com esses valores, foi estimado o erro médio de cada unidade experimental como sendo a média entre o erro da unidade considerada e os erros das unidades experimentais vizinhas (lado direito, lado esquerdo, da frente e do fundo) existentes (CARGNELUTTI FILHO et al., 2003). O valor do erro médio foi denominado de covariável para fins da análise de covariância, segundo o delineamento inteiramente casualizado (STEEL et al., 1997; STORCK et al., 2008), o que corresponde à aplicação do método de Papadakis.

Os valores da covariável também foram submetidos à análise de variância, segundo o modelo do delineamento blocos ao acaso, e testados quanto aos pressupostos da normalidade, aleatoriedade e homogeneidade das variâncias. A análise de covariância, com testes de hipóteses dos efeitos da covariável e de cultivar ajustados, foi realizada conforme STEEL et al. (1997) e com o cálculo das estimativas de amplitude entre médias de cultivares ajustadas, CV, D, DMS, IDF, $\mathrm{R}^{2}, \mathrm{AS}, \widehat{\rho}_{\mathrm{c}}$ e J $\left(\mathrm{R}^{2}\right)$.

Também foi estimada a eficiência relativa do uso da covariável em relação ao uso do delineamento blocos ao acaso e testadas as hipóteses dos pressupostos (normalidade, aleatoriedade e homogeneidade das variâncias para cultivares) referentes ao erro neste modelo. Para todos os cálculos, foi elaborado e compilado um programa específico com linguagem Pascal e os resultados aplicados numa planilha Excel para as estimativas de médias, amplitudes e coeficiente de variação.

\section{RESULTADOS E DISCUSSÃO}

Nos quatro anos em que os 48 ensaios foram executados em 11 localidades do sul do Brasil (Rio Grande do Sul, Santa Catarina e Paraná), a média de rendimento de grãos foi de $4,615 \mathrm{t} \mathrm{ha}^{-1}$, tendo variado entre 2,892 e 6,858t ha-1. Estes resultados são indicativos das variações do ambiente e/ou da lista de cultivares dos ensaios, pois cada cultivar é avaliada por um número variado de locais e de épocas de semeadura.

Em 25 ensaios (52\%), o efeito de blocos foi significativo (Tabela 2), provavelmente a variabilidade foi controlada com os bloqueamentos nas áreas experimentais. A variação entre as cultivares, significativa (valor-p $=0,05$ ) em $96 \%$ dos ensaios, e a ocorrência das falhas nos pressupostos (normalidade, aleatoriedade, homogeneidade da variância e aditividade do modelo matemático) (Tabela 2) está próxima ao encontrado nos estudos de 307 ensaios com milho (MARQUES et al., 2000), de 25 ensaios de competição de híbridos de milho (STORCK et al., 2010), em 226 ensaios de competição de genótipos de soja (STORCK et al., 2008) e em 26 ensaios de competição de cultivares de feijão (STORCK et al., 2011), não sendo motivo de restrição para a interpretação dos resultados nos ensaios de VCU de trigo.

Em todos os ensaios, a covariável (média do erro das unidades experimentais vizinhas) foi

Tabela 2 - Número de casos em que as diferentes hipóteses de nulidade foram rejeitadas, a $5 \%$ de probabilidade, para a análise de variância (Anova), para a covariável do método de Papadakis e para a análise pelo método de Papadakis, em 48 ensaios de competição de cultivares de trigo.

\begin{tabular}{llll}
\hline Hipótese & Anova & Covariável & Papadakis \\
\hline Blocos & $25(52 \%)$ & $35(73 \%)$ & - \\
Cultivares & $46(96 \%)$ & 0 & $48(100 \%)$ \\
Covariável & - & - & $48(100 \%)$ \\
Normalidade $(\mathrm{N})$ & $5(10 \%)$ & $12(25 \%)$ & $10(21 \%)$ \\
Aleatoriedade $(\mathrm{Al})$ & 0 & 0 & 0 \\
Homogeneidade $(\mathrm{H})$ & $1(2 \%)$ & $3(6 \%)$ & $1(2 \%)$ \\
Aditividade $(\mathrm{Ad})$ & $8(17 \%)$ & - & - \\
\hline
\end{tabular}


significativamente independente do efeito de cultivar (Tabela 2). Este fato, já observado em estudo de ensaios com soja, milho e feijão (STORCK et al., 2008, 2010 e 2011), indica que as diferenças entre os tratamentos independem dos valores da covariável, pré-requisito para proceder à análise de covariância e ajustar as médias de cultivares em função das médias da covariável (STEEL et al., 1997). Também, os pressupostos da aleatoriedade e da homogeneidade das variâncias foram adequados. No entanto, a falta de normalidade da covariável foi observada em 12 ensaios $(25 \%)$, condição semelhante ao observado $(16,4 \%)$ para ensaios com soja (STORCK et al., 2008).

Em relação à covariável, os blocos foram heterogêneos em $73 \%$ dos ensaios, superior ao observado (38,9\% dos ensaios) em soja (STORCK et al., 2008), 60\% em milho (STORCK et al., 2010) e $50 \%$ em feijão (STORCK et al., 2011). Esses resultados mostram que a maneira de calcular a covariável (média do erro entre as parcelas vizinhas) não absorve bem as diferenças entre blocos (lateralmente) nos ensaios de VCU de trigo, fato este que pode ser atribuído ao método mecânico de semeadura dos ensaios. Neste caso, os blocos são longos (sentido da semeadora) e estreitos (largura da semeadora).

A média da eficiência relativa (ER) do uso de blocos (quando heterogêneos) foi igual a 127,2\%
(Tabela 3), pois 52\% dos ensaios apresentaram blocos heterogêneos. Nos ensaios com soja, STORCK et al. (2008) observaram que a média da ER foi igual a $134,5 \%$ em apenas $28,8 \%$ dos ensaios com blocos heterogêneos, para ensaios com milho, a ER foi de $126 \%$ para $48 \%$ de ensaios com blocos heterogêneos (STORCK et al., 2010) e a ER foi de $173,8 \%$ para $46 \%$ de ensaios de feijão com blocos heterogêneos (STORCK et al., 2011). Devido aos valores da ER observados nas quatro culturas (trigo, milho, soja e feijão), em princípio, descartar o uso de blocos não seria recomendado.

A aplicação do método de Papadakis (parcelas vizinhas) aumentou de 96 para 100\% a proporção de ensaios com efeito de cultivares significativo (Tabela 2), sendo que, em $100 \%$ dos ensaios, o efeito da covariável também foi significativo. O uso do método de Papadakis melhorou todas as medidas de precisão experimental e reduziu a média do coeficiente de variação $(\mathrm{CV})$ de 7,5 para $5,1 \%$; a média da diferença mínima significativa entre as cultivares pelo teste de Tukey, de $1,08 \mathrm{t} \mathrm{ha}^{-1}$ para $0,75 \mathrm{tha}^{-1}$; a média da diferença mínima significativa entre as cultivares, pelo teste de Tukey, expresso em percentagem da média (DMS), de 24,5 para 16,8\%; e aumentou a média da acurácia seletiva (AS), de 0,90 para 0,95 ; a média do índice de diferenciação de

Tabela 3 - Média, amplitude (Ampl) e coeficiente de variação (CV) de diferentes estatísticas estimadas em ensaios de competição de cultivares de trigo (produtividade de grãos, $\mathrm{t} \mathrm{ha}^{-1}$ ), relacionado à aplicação do método de Papadakis.

\begin{tabular}{|c|c|c|c|c|c|c|}
\hline \multirow{2}{*}{ Estatísticas $^{(1)}$} & \multirow[b]{2}{*}{ Média } & \multirow[b]{2}{*}{ Ampl } & \multirow[b]{2}{*}{$\mathrm{CV}(\%)$} & \multirow[b]{2}{*}{ Média } & \multirow[b]{2}{*}{ Ampl } & \multirow[b]{2}{*}{$\mathrm{CV}(\%)$} \\
\hline & & & & & & \\
\hline $\mathrm{ER}(\%)$ & 114,7 & 145,4 & 20,5 & - & - & - \\
\hline Média $\left(\mathrm{t} \mathrm{ha}^{-1}\right)$ & 4,615 & 3,966 & 19,4 & - & - & - \\
\hline CVe $(\%)$ & 7,5 & 20,2 & 54,7 & 5,1 & 13,7 & 49,8 \\
\hline AS & 0,90 & 0,99 & 16,7 & 0,95 & 0,33 & 5,4 \\
\hline$h^{2}$ & 0,82 & 0,97 & 20,0 & 0,91 & 0,54 & 9,6 \\
\hline D Tukey & 1,08 & 2,50 & 42,4 & 0,75 & 1,70 & 40,2 \\
\hline Amplitude & 2,14 & 3,41 & 29,8 & 2,11 & 2,57 & 27,5 \\
\hline DMS (\%) & 24,5 & 71,3 & 55,7 & 16,8 & 48,9 & 51,2 \\
\hline IDF & 15,5 & 55,5 & 75,1 & 29,7 & 63,4 & 42,8 \\
\hline $\mathrm{R}^{2}(\%)$ & 86,1 & 48,3 & 10,3 & 92,4 & 34,1 & 6,4 \\
\hline Beta & - & - & - & 1,437 & 0,523 & 8,9 \\
\hline ER P (\%) & - & - & - & 123,1 & 160,5 & 32,6 \\
\hline$\hat{\rho}_{g}$ & 0,649 & 0,927 & 28,0 & 0,796 & 0,742 & 16,7 \\
\hline $\mathrm{J}\left(\mathrm{R}^{2}=90 \%\right)$ & 6,1 & 62,4 & 148,4 & 3,0 & 33,3 & 167,0 \\
\hline
\end{tabular}

${ }^{(1)}$ ER- eficiência relativa dos ensaios com efeito de blocos significativos; CVe - coeficiente de variação do erro experimental; AS - acurácia seletiva; $h^{2}$ - coeficiente de herdabilidade; D Tukey - diferença mínima significativa pelo teste de Tukey (valor-p=0,05); amplitude entre médias; DMS - D Tukey em percentagem da média; IDF - índice de diferenciação de Fasoulas; $\mathrm{R}^{2}$ - coeficiente de determinação do modelo; Beta - efeito da covariável, coeficiente de regressão linear; ER P - eficiência relativa do uso do método de Papadakis; $\widehat{\rho}_{\mathrm{c}}$ - coeficiente de repetibilidade; $\mathrm{J}\left(\mathrm{R}^{2}=90 \%\right)$ - número de repetições associado à determinação ou à precisão de $90 \%$. 
Fasoulas (IDF), de 15,5 para 29,7; e o coeficiente de determinação do modelo $\left(\mathrm{R}^{2}\right)$, de $86,1 \%$ para $92,4 \%$ (Tabela 3). Respostas com ganhos semelhantes, para essas medidas de precisão, foram obtidas para 226 ensaios com soja (STORCK et al., 2008), para 25 ensaios com híbridos de milho (STORCK et al., 2010) e para 26 ensaios com cultivares de feijão (STORCK et al., 2011). Estes dados confirmam que o uso do método de Papadakis melhora a qualidade dos resultados das análises dos ensaios e, por isso, a sua utilização deve ser considerada pelos melhoristas de plantas na avaliação de genótipos.

Particularmente, a acurácia seletiva (AS), medida de precisão experimental adequada para os ensaios com milho (CARGNELUTTI FILHO \& STORCK, 2009), feijão e soja (CARGNELUTTI FILHO et al., 2009), mostrou que, em $75 \%$ dos ensaios, sem o uso do método de Papadakis (parcelas vizinhas), a precisão foi muito alta ( $\mathrm{AS} \geq 0,90)$. Já, com o uso deste, precisão muito alta foi observada em $94 \%$ dos ensaios, o que é uma evidência da importância do uso deste método para a melhoria da precisão experimental. $\mathrm{O}$ uso do método de Papadakis, neste estudo e nos de soja, milho e feijão, proporcionou aumento da média da acurácia seletiva e redução da amplitude de variação e do coeficiente de variação dessa medida de precisão, isto é, a precisão experimental ficou mais homogênea e mais alta.

Em média, o uso do método proposto foi $23,1 \%$ mais eficiente do que o uso do modelo com delineamento blocos ao acaso, pois a eficiência relativa (ER P, Tabela 3) foi igual a $123,1 \%$, com amplitude de $160,5 \%$ e CV de $32,6 \%$. Outro aspecto favorável ao método de Papadakis é que os pressupostos do erro, com o modelo da análise de covariância, foram violados em proporções semelhantes ao da aplicação do modelo do delineamento de blocos ao acaso (Tabela 2). Resultados favoráveis ao uso do método de Papadakis também foram relatados para aplicação no melhoramento genético de feijão e de milho (SOUZA et al., 2000; COSTA et al., 2005). Por outro lado, CANDIDO et al. (2009) concluíram que o método de Papadakis não melhorou a precisão experimental, bem como as consequências no ordenamento de genótipos de cana-de-açúcar em termos de potencial produtivo (colmos). Possivelmente, por se tratar de característica avaliada que não depende tanto da interação com as condições ambientais no momento da reprodução e produção dos grãos (soja, milho, feijão, trigo).

A média dos coeficientes de regressão linear (efeito da covariável, Beta) foi igual a 1,437, com amplitude de 0,523 (Tabela 3), o que evidencia que os valores de rendimento de grãos puderam ser ajustados significativamente, em $100 \%$ dos ensaios, para mais ou para menos, com intensidades variadas nos diferentes ensaios. Para cada unidade a mais na covariável (média do erro das parcelas vizinhas nas $\mathrm{J}$ repetições), o valor da média da cultivar é acrescido em $43,7 \%$ (multiplicado por 1,437), valor este semelhante ao obtido para ensaios de soja $(44,2 \%)$ (STORCK et al., 2008), maior que o valor de $34,3 \%$ obtido com milho (STORCK et al., 2010) e maior que o valor de $28,7 \%$ obtido com feijão (STORCK et al., 2011). Nas quatro culturas, observam-se ganhos importantes na precisão experimental com o uso do método de Papadakis.

$\mathrm{Na}$ análise de repetibilidade, o valor médio do coeficiente de correlação intraclasse $\left(\hat{\rho}_{\mathrm{c}}\right)$ estimado no método Papadakis foi de 0,796 , sendo que a redução do número de repetições necessário para uma dada precisão foi expressiva. Isso significa dizer que, ao aplicar o método de Papadakis, foi necessário, em média, apenas a metade do número de repetições para predizer o valor real dos indivíduos (cultivares), com base na determinação ou na precisão pré-estabelecida $\left(\mathrm{R}^{2}=0,90\right)$, se comparado com a análise usual (sem a aplicação do método de Papadakis) (Tabela 3). Esta redução do número de repetições foi a mesma obtida em feijão (STORCK et al., 2011) e foi menor do que a obtida em estudo com a cultura da soja e do milho com, respectivamente, um quarto e um terço das repetições (STORCK et al., 2008 e 2010). Em todos os ensaios de trigo utilizados no estudo, foram usadas três repetições, valor inferior as seis repetições necessárias para constatar diferenças existentes entre cultivares, com uma confiança de $90 \%$. No entanto, para uma mesma confiança, usando o método de Papadakis, as três repetições são suficientes e a determinação ou precisão seria ainda um pouco superior $\left(\mathrm{R}^{2}=0,921\right)$.

\section{CONCLUSÃO}

A análise de rendimento de grãos dos ensaios de competição de cultivares de trigo por meio do método de Papadakis mantém adequados os pressupostos de normalidade, aleatoriedade, homogeneidade.

O método de Papadakis melhorou os índices das medidas de precisão experimental e reduziu o número de repetições necessário para a predição do desempenho de cultivares de trigo, com um mesmo grau de precisão, quando se avaliou o rendimento de grãos. 


\section{AGRADECIMENTOS}

Ao Conselho Nacional de Desenvolvimento Científico e Tecnológico $(\mathrm{CNPq})$, pela concessão de bolsas de produtividade em pesquisa e a Coordenação de Aperfeiçoamento de Pessoal de Nível Superior (CAPES) (Edital 6/2012, PVNS). À Equipe de experimentação da Embrapa Trigo, Adriano Boeira, Eurico Bocalon dos Santos, Clóvis Roberto Signori e Sérgio Adriani Zanchett.

\section{REFERÊNCIAS}

CANDIDO, L.S. et al. Análise de vizinhança na avaliação de genótipos de cana-de-acucar. Pesquisa Agropecuária Brasileira v.44, p.1304-1311, 2009. Disponível em: <http://www.scielo.br/ pdf/pab/v44n10/v44n10a14.pdf $>$. Acesso em: 11 ago. 2013. doi: 10.1590/S0100-204X2009001000014.

CARGNELUTTI FILHO, A.; STORCK, L. Estatísticas de avaliação da precisão experimental em ensaios de cultivares de milho. Pesquisa Agropecuária Brasileira, v.42, p.1724, 2007. Disponível em: <http://www.scielo.br/pdf/pab/ v42n1/03.pdf>. Acesso em: 11 ago. 2013. doi: 10.1590/S0100204X2007000100003.

CARGNELUTTI FILHO, A.; STORCK, L. Medidas do grau de precisão experimental em ensaios de competição de cultivares de milho. Pesquisa Agropecuária Brasileira, v.44, p.111-117, 2009. Disponível em: <http://www.scielo.br/pdf/pab/v44n2/ v44n02a01.pdf>. Acesso em: 11 ago. 2013. doi: 10.1590/S0100204X2009000200001.

CARGNELUTTI FILHO, A. et al. Ajustes de quadrado médio do erro em ensaios de competição de cultivares de milho pelo método de Papadakis. Pesquisa Agropecuária Brasileira, v.38, p.467473, 2003. Disponível em: <http://www.scielo.br/pdf/pab/v38n4/ a04v38n4.pdf $>$. Acesso em: 11 ago. 2013. doi: 10.1590/S0100$204 \times 2003000400004$.

CARGNELUTTI FILHO, A. et al. Medidas da precisão experimental em ensaios com genótipos de feijão e de soja. Pesquisa Agropecuária Brasileira, v.44, p.1225-1231, 2009. Disponível em: <http://www.scielo.br/pdf/pab/v44n10/ v44n10a03.pdf>. Acesso em: 11 ago. 2013. doi: 10.1590/S0100204X2009001000003.

COSTA, J.R. et al. Análise espacial e de vizinhança no melhoramento genético de plantas. Pesquisa Agropecuária Brasileira, v.40, p.1073-1079, 2005. Disponível em: <http:// www.scielo.br/pdf/pab/v40n11/a04v4011.pdf>. Acesso em: 11 ago. 2013. doi: 10.1590/S0100-204X2005001100004.

CRUZ, C.D.; REGAZZI, A.J. Modelos biométricos aplicados ao melhoramento genético. 2.ed. Viçosa: UFV, 1997. 390p.

FASOULAS, A.C. Rating cultivars and trials in applied plant breeding. Euphytica, v.32, p.939-943, 1983. Disponível em: $<$ http://link.springer.com/article/10.1007\%2FBF00042176>. Acesso em: 11 ago. 2013.
HENRIQUES NETO, D. et al. Tamanho de parcelas em experimentos com trigo irrigado sob plantio direto e convencional. Pesquisa Agropecuária Brasileira, v.39, p.517-524, 2004. Disponível em: <http:/www.scielo.br/pdf/pab/v39n6/v39n6a01. pdf $>$. Acesso em: 11 ago. 2013. doi: 10.1590/S0100204X2004000600001.

LORENTZ, L.H. et al. Tamanho de parcela e precisão experimental em ensaios com trigo em plantio direto. Científica, v.35, p.129135, 2007. Disponível em: < http://www.cientifica.org.br/index. php/cientifica/article/view/191/112>. Acesso em: 11 ago. 2013.

MARQUES, D.G. et al. Qualidade dos ensaios de competição de cultivares de milho no Estado do Rio Grande do Sul. Ciência Rural, v.30, p.381-385, 2000. Disponível em: $<$ http://www.scielo. br/pdf/cr/v30n3/a01v30n3.pdf $>$. Acesso em: 11 ago. 2013. doi: 10.1590/S0103-84782000000300001.

RAMALHO, M.A.P. et al. Experimentação em genética e melhoramento de plantas. 2.ed. Lavras: UFLA, 2005. 322p.

RESENDE, M.D.V.; DUARTE, J.B. Precisão e controle de qualidade em experimentos de avaliação de cultivares. Pesquisa Agropecuária Tropical, v.37, p.182-194, 2007. Disponível em: $<$ http://www.revistas.ufg.br/index.php/pat/article/view/1867/1773>. Acesso em: 11 ago. 2013.

SNEDECOR, G.W.; COCHRAN, W.G. Statistical methods. 8.ed. Ames: Iowa State University, 1989. 503p.

SOUZA, E.A. et al. Alternativas experimentais na avaliação de famílias em programas de melhoramento genético do feijoeiro. Pesquisa Agropecuária Brasileira, v.35, p.1765-1771, 2000. Disponível em: <http:/www.scielo.br/pdf/pab/v35n9/ v35n9a09.pdf>. Acesso em: 11 ago. 2013. doi: 10.1590/S0100204X2000000900009.

SPRENT, P.; SMEETON, N.C. Applied nonparametric statistical methods. 4.ed. Boca Raton: Chapman \& Hall, 2007. 530p.

STEEL, R.G.D. et al. Principles and procedures of statistics: a biometrical approach. 3.ed. New York: McGraw Hill Book, 1997. $666 p$.

STORCK, L. et al. Experimental precision in corn trials using the Papadakis method. Ciência e Agrotecnologia, v.34, p.14581464, 2010. Disponível em: <http://www.scielo.br/pdf/cagro/ v34n6/15.pdf>. Acesso em: 11 ago. 2013. doi: 10.1590/S141370542010000600015 .

STORCK, L. et al. Precisão experimental de ensaios de feijão analisada pelo método de Papadakis. Pesquisa Agropecuária Brasileira, v.46, p.798-804, 2011. Disponível em: < http://www. scielo.br/pdf/pab/v46n8/03.pdf>. Acesso em: 11 ago. 2013. doi: 10.1590/S0100-204X2011000800003.

STORCK, L. et al. Utilização do método de Papadakis na melhoria da qualidade experimental de ensaios com soja. Pesquisa Agropecuária Brasileira, v.43, p.581-587, 2008. Disponível em: $<$ http://www.scielo.br/pdf/pab/v43n5/a05v43n5.pdf >. Acesso em: 11 ago. 2013. doi: 10.1590/S0100-204X2008000500005. 